\title{
Deconfinement and chiral restoration in non-local PNJL models at zero and imaginary chemical potential
}

\author{
V. Pagura ${ }^{\mathrm{a}, \mathrm{b}}$, D. Gómez Dumm ${ }^{\mathrm{b}, \mathrm{c}, *}$, N.N. Scoccola ${ }^{\mathrm{a}, \mathrm{b}, \mathrm{d}}$ \\ a Physics Department, Comisión Nacional de Energía Atómica, Av. Libertador 8250, 1429 Buenos Aires, Argentina \\ b CONICET, Rivadavia 1917, 1033 Buenos Aires, Argentina \\ c IFLP, CONICET - Dpto. de Física, Universidad Nacional de La Plata, C.C. 67, 1900 La Plata, Argentina \\ d Universidad Favaloro, Solís 453, 1078 Buenos Aires, Argentina
}

\section{A R T I C L E I N F O}

\section{Article history:}

Received 11 May 2011

Received in revised form 1 November 2011

Accepted 30 November 2011

Available online 2 December 2011

Editor: J.-P. Blaizot

\begin{abstract}
A B S T R A C T
We study the deconfinement and chiral restoration transitions in the context of non-local PNJL models, considering the impact of the presence of dynamical quarks on the scale parameter appearing in the Polyakov potential. We show that the corresponding critical temperatures are naturally entangled for both zero and imaginary chemical potential, in good agreement with lattice QCD results. We also analyze the Roberge-Weiss transition, which is found to be first order at the associated endpoint.
\end{abstract}

(c) 2011 Elsevier B.V. All rights reserved.
The detailed understanding of the behavior of strongly interacting matter at finite temperature and baryon density represents an issue of great interest in particle physics [1]. From the theoretical point of view, this problem can be addressed through lattice QCD calculations [2-4], which have been significantly improved in the last years. However, this ab initio approach is not yet able to provide a full understanding of the QCD phase diagram. One wellknown difficulty is given by the so-called sign problem, which arises when dealing with finite real chemical potentials. Thus, it is worth to develop alternative approaches, such as the study of effective models that show consistency with lattice QCD results and can be extrapolated into regions not accessible by lattice techniques. One of these effective theories, proposed quite recently, is the so-called Polyakov-Nambu-Jona-Lasinio (PNJL) model [5-11], an extension of the well-known NJL model [12] in which quarks are coupled to the Polyakov loop (PL), providing a common framework to study both the chiral and deconfinement transitions. As a further improvement over the (local) PNJL model, extensions that include covariant non-local quark interactions have also been considered [13-15]. The non-local character of the interactions arises naturally in the context of several successful approaches to lowenergy quark dynamics, and leads to a momentum dependence in the quark propagator that can be made consistent [16] with lattice results. It has been shown [17-20] that non-local models provide a satisfactory description of hadron properties at zero temperature and density. Moreover, it has been found that, under certain

\footnotetext{
* Corresponding author at: CONICET, Rivadavia 1917, 1033 Buenos Aires, Argentina.

E-mail address: dumm@fisica.unlp.edu.ar (D. Gómez Dumm).
}

conditions, it is possible to derive the main features of non-local PNJL models starting directly from QCD [21]. Related PolyakovDyson-Schwinger equation models have also been recently considered [22].

The aim of the present work is to analyze the relation between the deconfinement and chiral restoration transitions at both zero and imaginary chemical potential $\mu$ in the context of non-local chiral quark models. One of the problems of the standard (local) PNJL model is that once the PL potential is adjusted to reproduce the pure gauge lattice QCD results, it is found [8] that the critical temperature for the chiral and deconfinement transitions at vanishing chemical potential, $T_{c} \approx 220 \mathrm{MeV}$, is somewhat too high in comparison with the presently most accepted lattice result, namely $T_{C}=173(8) \mathrm{MeV}$ for two light flavors [4]. A solution to this difficulty follows from the observation made in the context of the Polyakov quark-meson model [23], where it is claimed that in the presence of dynamical quarks one should decrease the parameter $T_{0}$ which sets the scale of the PL potential. However, in contradiction to lattice results, in the PNJL model this sort of rescaling leads to a rather noticeable splitting between the deconfinement and chiral restoration temperatures. This splitting can be avoided only after the inclusion of extra eight-quark interactions [24], or by assuming that the quark-quark coupling constant is some ad hoc function of the Polyakov loop [25]. Here we show that in the case of the non-local SU(2) PNJL model the critical temperature can be made naturally compatible with lattice QCD estimates, without spoiling the entanglement between deconfinement and chiral restoration transition temperatures even for imaginary chemical potential. It should be stressed that the extension to imaginary chemical potential deserves significant theoretical interest, since lattice calculations [26-28] become free of the sign 
problem and the corresponding results can be compared with effective model predictions. Moreover, the behavior in the region of imaginary chemical potential is expected to have implications on the QCD phase diagram at finite real values of $\mu$. Lattice calculations, as well as analyzes based on the exact renormalization group equations [29], suggest a close relation between the deconfinement and chiral restoration transitions for imaginary chemical potentials. Thus, we extend our study of these transitions to the region of imaginary $\mu$, where we also analyze the characteristics of the socalled Roberge-Weiss (RW) transition [30], in particular, at the RW endpoint.

Let us briefly describe the model under consideration, namely a non-local $\mathrm{SU}(2)$ chiral quark theory that includes couplings to a background color gauge field. The Euclidean effective action is given by [31]

$$
\begin{aligned}
S_{E}= & \int d^{4} x\left\{\bar{\psi}(x)\left(-i \gamma_{\mu} D_{\mu}+\hat{m}\right) \psi(x)\right. \\
& \left.-\frac{G_{S}}{2}\left[j_{a}(x) j_{a}(x)-j_{P}(x) j_{P}(x)\right]+\mathcal{U}(\Phi[A(x)])\right\},
\end{aligned}
$$

where $\psi$ is the $N_{f}=2$ fermion doublet $\psi \equiv(u, d)^{T}, \hat{m}=m_{q} \mathbb{1}_{2 \times 2}$ is the current quark mass matrix in the isospin limit, and $D_{\mu} \equiv$ $\partial_{\mu}-i A_{\mu}$ is a covariant derivative, $A_{\mu}$ being color gauge fields. The non-local currents $j_{a}(x), j_{P}(x)$ are given by

$$
\begin{aligned}
& j_{a}(x)=\int d^{4} z \mathcal{G}(z) \bar{\psi}\left(x+\frac{z}{2}\right) \Gamma_{a} \psi\left(x-\frac{z}{2}\right), \\
& j_{P}(x)=\int d^{4} z \mathcal{F}(z) \bar{\psi}\left(x+\frac{z}{2}\right) \frac{i \overleftrightarrow{\not}}{2 \kappa_{p}} \psi\left(x-\frac{z}{2}\right),
\end{aligned}
$$

where $\Gamma_{a}=\left(\mathbb{1}, i \gamma_{5} \vec{\tau}\right)$, and the functions $\mathcal{F}(z)$ and $\mathcal{G}(z)$ are nonlocal form factors that characterize the interactions. Notice that even if we take for convenience the same coupling parameter $G_{S}$ for both interaction terms, the relative strength between them is controlled by the mass parameter $\kappa_{p}$.

To proceed we perform a standard bosonization of the theory, introducing bosonic fields $\sigma_{1,2}(x)$ and $\pi_{a}(x)$, and integrating out the quark fields. We will work within the mean field approximation (MFA), in which these bosonic fields are replaced by their vacuum expectation values $\sigma_{1,2}$ and $\pi_{a}=0$. Since we are interested in studying the characteristics of the chiral phase transition, we extend the bosonized effective action to finite temperature $T$ and chemical potential $\mu$. This can be done by using the Matsubara formalism. Concerning the gauge fields $A_{\mu}$, we assume that quarks move on a constant background field $\phi=A_{4}=$ $i A_{0}=i g \delta_{\mu 0} G_{a}^{\mu} \lambda^{a} / 2$, where $G_{a}^{\mu}$ are the $\mathrm{SU}(3)$ color gauge fields. Then the traced Polyakov loop, which in the infinite quark mass limit can be taken as order parameter of confinement, is given by $\Phi=\frac{1}{3} \operatorname{Tr} \exp (i \phi / T)$. We will work in the so-called Polyakov gauge, in which the matrix $\phi$ is given a diagonal representation $\phi=\phi_{3} \lambda_{3}+\phi_{8} \lambda_{8}$. This leaves only two independent variables, $\phi_{3}$ and $\phi_{8}$. The mean field traced Polyakov loop reads then

$\Phi=\frac{1}{3}\left[\exp \left(-\frac{2 i}{\sqrt{3}} \frac{\phi_{8}}{T}\right)+2 \exp \left(\frac{i}{\sqrt{3}} \frac{\phi_{8}}{T}\right) \cos \left(\frac{\phi_{3}}{T}\right)\right]$.

Within this framework the mean field thermodynamical potential $\Omega^{\mathrm{MFA}}$ at finite temperature and arbitrary (in general, complex) chemical potential is given by

$$
\begin{aligned}
\Omega^{\mathrm{MFA}}= & -4 T \sum_{c=r, g, b} \sum_{n=-\infty}^{\infty} \int \frac{d^{3} \vec{p}}{(2 \pi)^{3}} \ln \left[\frac{\left(\rho_{n, \vec{p}}^{c}\right)^{2}+M^{2}\left(\rho_{n, \vec{p}}^{c}\right)}{Z^{2}\left(\rho_{n, \vec{p}}^{c}\right)}\right] \\
& +\frac{\sigma_{1}^{2}+\kappa_{p}^{2} \sigma_{2}^{2}}{2 G_{S}}+\mathcal{U}\left(\Phi, \Phi^{*}, T\right) .
\end{aligned}
$$

Here, $M(p)$ and $Z(p)$ are given by

$M(p)=Z(p)\left[m_{q}+\sigma_{1} g(p)\right], \quad Z(p)=\left[1-\sigma_{2} f(p)\right]^{-1}$,

where $g(p)$ and $f(p)$ are Fourier transforms of $\mathcal{G}(z)$ and $\mathcal{F}(z)$. We have also defined

$\left(\rho_{n, \vec{p}}^{c}\right)^{2}=\left[(2 n+1) \pi T-i \mu+\phi_{c}\right]^{2}+\vec{p}^{2}$,

where the quantities $\phi_{c}$ are given by the relation $\phi=\operatorname{diag}\left(\phi_{r}, \phi_{g}\right.$, $\left.\phi_{b}\right)$, i.e. $\phi_{r}=\phi_{3}+\phi_{8} / \sqrt{3}, \phi_{g}=-\phi_{3}+\phi_{8} / \sqrt{3}, \phi_{b}=-2 \phi_{8} / \sqrt{3}$.

To proceed we need to specify the explicit form of the Polyakov loop effective potential $\mathcal{U}\left(\Phi, \Phi^{*}, T\right)$. Following Ref. [9] we take

$$
\begin{aligned}
\mathcal{U}\left(\Phi, \Phi^{*}, T\right)= & \left\{-\frac{1}{2} a(T) \Phi \Phi^{*}+b(T) \ln \left[1-6 \Phi \Phi^{*}+4 \Phi^{3}\right.\right. \\
& \left.\left.+4\left(\Phi^{*}\right)^{3}-3\left(\Phi \Phi^{*}\right)^{2}\right]\right\} T^{4},
\end{aligned}
$$

where the coefficients are parameterized as

$a(T)=a_{0}+a_{1}\left(\frac{T_{0}}{T}\right)+a_{2}\left(\frac{T_{0}}{T}\right)^{2}, \quad b(T)=b_{3}\left(\frac{T_{0}}{T}\right)^{3}$.

The values of $a_{i}$ and $b_{3}$ are fitted [9] to lattice QCD results, which in absence of dynamical quarks lead to a deconfinement temperature $T_{0} \approx 270 \mathrm{MeV}$. However, as mentioned above, it has been argued [23] that in the presence of light dynamical quarks this value has to be modified accordingly, e.g. $T_{0} \simeq 208 \mathrm{MeV}$ for $N_{f}=2$ and $T_{0} \simeq 180 \mathrm{MeV}$ for $N_{f}=3$. Effects of this change in $T_{0}$ will be discussed below. In addition, it is seen that $\Omega^{\mathrm{MFA}}$ turns out to be divergent, thus it has to be regularized. Here we use the same prescription as e.g. in Ref. [32], namely

$\Omega_{\mathrm{reg}}^{\mathrm{MFA}}=\Omega^{\mathrm{MFA}}-\Omega^{\text {free }}+\Omega_{\mathrm{reg}}^{\text {free }}+\Omega_{0}$,

where $\Omega^{\text {free }}$ is obtained from Eq. (4) by setting $\sigma_{1}=\sigma_{2}=0$, and $\Omega_{\text {reg }}^{\text {free }}$ is the regularized expression for the quark thermodynamical potential in the absence of fermion interactions:

$$
\begin{aligned}
\Omega_{\mathrm{reg}}^{\text {free }}= & -4 T \int \frac{d^{3} \vec{p}}{(2 \pi)^{3}} \\
& \times \sum_{c=r, g, b} \sum_{s= \pm 1} \operatorname{Re} \ln \left\{1+\exp \left[-\frac{\epsilon_{p}+s\left(\mu+i \phi_{c}\right)}{T}\right]\right\}
\end{aligned}
$$

with $\epsilon_{p}=\sqrt{\vec{p}^{2}+m_{q}^{2}}$. The last term in Eq. (9) is just a constant fixed by the condition that $\Omega_{\mathrm{reg}}^{\mathrm{MFA}}$ vanishes at $T=\mu=0$.

The mean field values $\sigma_{1,2}$ and $\phi_{3,8}$ can be obtained from a set of four coupled "gap" equations that follow from the minimization of the regularized thermodynamical potential,

$\frac{\partial \Omega_{\mathrm{reg}}^{\mathrm{MFA}}}{\partial \sigma_{1}}=\frac{\partial \Omega_{\mathrm{reg}}^{\mathrm{MFA}}}{\partial \sigma_{2}}=\frac{\partial \Omega_{\mathrm{reg}}^{\mathrm{MFA}}}{\partial \phi_{3}}=\frac{\partial \Omega_{\mathrm{reg}}^{\mathrm{MFA}}}{\partial \phi_{8}}=0$.

Once the mean field values are obtained, the behavior of other relevant quantities as functions of the temperature and chemical potential can be determined. We concentrate in particular in the chiral quark condensate $\langle\bar{q} q\rangle=\partial \Omega_{\mathrm{reg}}^{\mathrm{MFA}} / \partial m_{q}$, which together with the modulus of the Polyakov loop $|\Phi|$ will be taken as order parameters of the chiral restoration and deconfinement transitions, respectively. For simplicity, the associated susceptibilities will be defined as $\chi_{\text {cond }}=d\langle\bar{q} q\rangle / d T$ and $\chi_{P L}=$ $d|\Phi| / d T$.

In order to fully specify the model under consideration we have to fix the model parameters as well as the form factors $g(q)$ and $f(q)$ that characterize the non-local interactions. Here we consider 

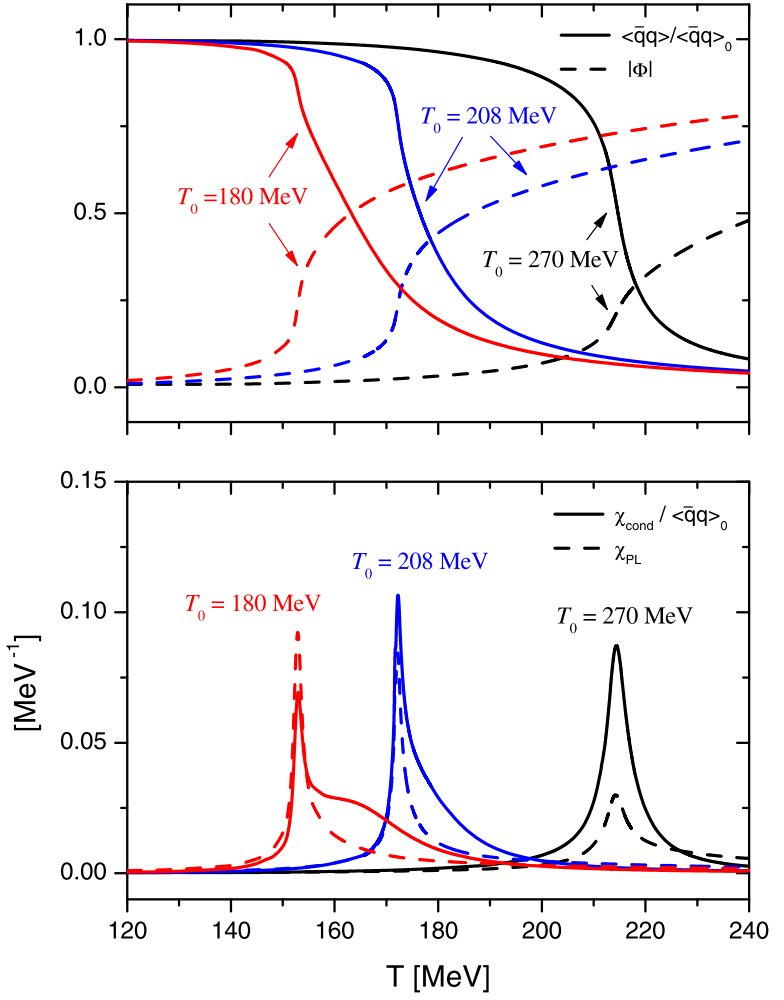

Fig. 1. Order parameters for the deconfinement and chiral restoration transitions (upper panel) and the corresponding susceptibilities (lower panel) as functions of $T$ for three characteristic values of $T_{0}$, namely $T_{0}=270,208$ and $180 \mathrm{MeV}$. Curves correspond to set $\mathrm{C}$.

three parameter sets A, B and C, which have been introduced in Ref. [16]. Set A corresponds to the relatively simple case in which there is no wave function renormalization (WFR) of the quark propagator, i.e. $f(p)=0, Z(p)=1$, and $g(p)$ has a Gaussian behavior, $g(p)=\exp \left(-p^{2} / \Lambda_{0}^{2}\right)$. In set B we consider a more general case that includes quark WFR, taking also an exponential shape for the corresponding form factor, $f(p)=\exp \left(-p^{2} / \Lambda_{1}^{2}\right)$. Finally, in set $\mathrm{C}$ we take Lorentzian-like form factors, chosen in such a way that one can well reproduce the momentum dependence of mass and WFR functions obtained in lattice calculations. The parameter values for sets A, B and C, together with the corresponding predictions for several meson properties, can be found in Ref. [16].

Let us analyze the deconfinement and chiral transitions at vanishing chemical potential in the framework of the model presented above. Taking $T_{0}$ as a parameter, and solving numerically Eqs. (11) for sets $A, B$ and $C$, it is found that both the deconfinement and chiral restoration temperatures are coincident in a wide range of values of $T_{0}$. This is illustrated in Fig. 1, where we show the behavior of the relevant order parameters and the corresponding susceptibilities for the lattice inspired parameterization set $\mathrm{C}$. We consider three characteristic values $T_{0}=270,208$ and $180 \mathrm{MeV}$, corresponding to the presence of 0,2 and 3 dynamical fermions, respectively [23]. It is clear that for this set both transitions are crossover-like, and they occur at basically the same critical temperature, as it is indicated by the peaks of the corresponding susceptibilities. It is interesting to notice that, in agreement with recent lattice results with physical quark masses [33], the rise of the Polyakov loop with the temperature appears to be smoother than that obtained in local models. One might notice that as long as $T_{0}$ decreases $\chi_{\text {cond }}$ tends to become asymmetric around $T_{c}$, being somewhat broader on the high temperature side. Though this could be considered as an indication that for smaller values of $T_{0}$

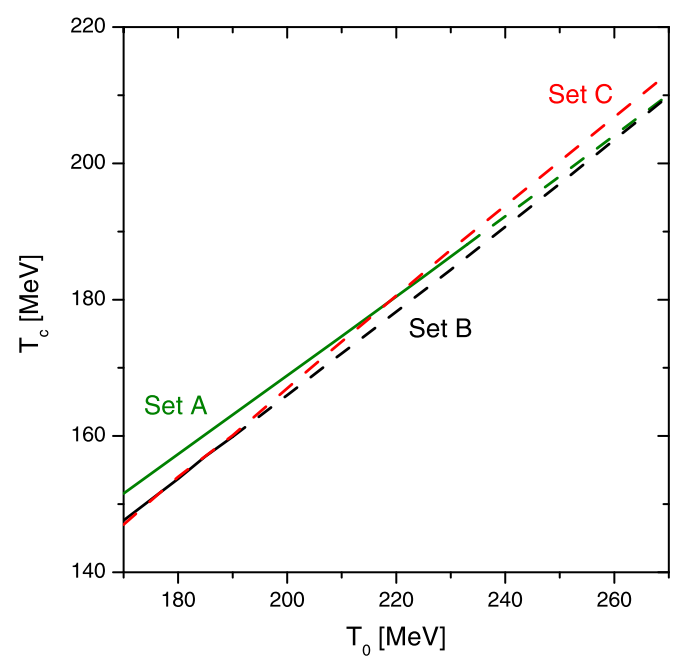

Fig. 2. Critical temperature as a function of $T_{0}$ for our parameter sets $A, B$ and $C$. Solid and dashed lines stand for first order and crossover-like transitions, respectively. Note that below $T_{0} \sim 190 \mathrm{MeV}$ the curves for sets B and $\mathrm{C}$ almost overlap. However, while for set $\mathrm{B}$ the transition in that region is first order (solid line), for set $\mathrm{C}$ it is crossover-like (dashed line).

chiral symmetry is restored at a slightly higher temperature, even at $T_{0}=180 \mathrm{MeV}$ the splitting between the main peak and what might be considered as a second broad peak is less than $10 \mathrm{MeV}$. In addition, it is worth to point out that although the coincidence of the deconfinement and chiral restoration critical temperatures holds for all three parameter sets A, B and C, the character of the transitions may be different from one another. This is shown in Fig. 2, where we plot the values of the critical temperatures as functions of $T_{0}$ for sets $A, B$ and $C$. We see that for set $A$, which does not include WFR, the transition becomes a first order one for values of $T_{0}$ below $\simeq 235 \mathrm{MeV}$. On the other hand, for the exponential parameterization with WFR, set $B$, this happens at a lower value $T_{0} \simeq 180 \mathrm{MeV}$. Finally, as already mentioned, for the lattice inspired parameterization set $C$ the transitions are crossover-like for all considered values of $T_{0}$. It should be stressed that for $T_{0}=208 \mathrm{MeV}$ (corresponding to our $N_{f}=2$ model) the resulting critical temperatures are in good agreement with lattice QCD estimates. Indeed, we get $T_{C}(0)=173,171$ and $173 \mathrm{MeV}$ for sets $A, B$ and $C$, respectively. It is important to remark that the nature of deconfinement and chiral restoration transitions for two light flavors in lattice QCD is still under debate. While most studies [34-38] favor a second order transition in the chiral limit, there are also claims for a first order transition $[39,40]$. Given that in the context of the present non-local model the parameterizations that include WFR appear to be more realistic, the second order scenario turns out to be preferred. It is also worth to mention that in the standard Pisarski-Wilczek scenario the order of the phase transition for two flavor QCD is closely related with the fate of the anomaly at the transition point [41].

We consider now the situation at nonzero imaginary chemical potential. As it is well known, Roberge and Weiss found [30] that the thermodynamical potential of QCD in presence of an imaginary chemical potential $\mu=i \theta T$ is a periodic function of $\theta$ with period $2 \pi / 3$. This means that $Q C D$ is invariant under a combination of a $Z_{3}$ transformation of the quark and gauge fields and a shift $\theta \rightarrow \theta+2 k \pi / 3$, with integer $k$, in the chemical potential. Recently, it has been shown that this so-called extended $Z_{3}$ transformation is also a symmetry of the local Polyakov-NambuJona-Lasinio model [42]. Indeed, in the context of this model the thermodynamical potential is invariant under the transformations 
$\Phi(\theta) \rightarrow \Phi(\theta) \exp (-i 2 k \pi / 3)$

$\Phi^{*}(\theta) \rightarrow \Phi^{*}(\theta) \exp (i 2 k \pi / 3), \quad \theta \rightarrow \theta+2 k \pi / 3$.

The RW periodicity is a remnant of the $Z_{3}$ symmetry in the pure gauge limit. In QCD with dynamical quarks, if the temperature is larger than a certain value $T_{R W}$ it can be seen that three $Z_{3}$ vacua appear. These vacua can be classified through their Polyakov loop phases, given by $\varphi, \varphi+2 \pi / 3$ and $\varphi+4 \pi / 3$. Roberge and Weiss showed that for $T>T_{R W}$ there is a first order phase transition at $\theta=\pi / 3 \bmod 2 \pi / 3$, in which the vacuum jumps to one of its $Z_{3}$ images. This is known as the "Roberge-Weiss transition", and the point at the end of the RW transition line in the $(T, \theta)$ plane, i.e. $(T, \theta)=\left(T_{R W}, 2 \pi / 3\right)$, is known as the "RW endpoint". The order of the RW transition at the RW endpoint has been subject of considerable interest recently in the framework of lattice QCD [43-46] due to the implications it might have on the QCD phase diagram a finite real $\mu$. According to lattice calculations, it appears that for two light flavors the RW endpoint is first order for realistically small values of the current quark mass. Following these considerations it is important to check whether the thermodynamical potential of the non-local PNJL models studied in this work does respect the extended $Z_{3}$ symmetry. In fact, it is easy to show that this is the case. The last two terms in Eq. (4) are obviously invariant under the transformations in Eq. (12), whereas to check the invariance of the first term it is convenient to write these transformations in the equivalent way

$$
\begin{aligned}
& \phi_{3}(\theta) \rightarrow \phi_{3}(\theta), \quad \phi_{8}(\theta) \rightarrow \phi_{8}(\theta)-2 k \pi T / \sqrt{3}, \\
& \theta \rightarrow \theta+2 k \pi / 3 .
\end{aligned}
$$

Thus it can be easily proven that any sum of the form $\sum_{c=r, g, b} F\left[\left(\rho_{n, \vec{p}}^{c}\right)^{2}\right]$, where $F$ is an arbitrary function, turns out to be invariant under the extended $Z_{3}$ transformations. The invariance of the terms introduced in the regularization procedure [cf. Eq. (9)] can be shown in the same way.

Having checked that our non-local PNJL models possess the extended $Z_{3}$ invariance we turn now to the results of the numerical analysis of the behavior of the different order parameters as functions of $T$ and $\theta$ using the value $T_{0}=208 \mathrm{MeV}$ corresponding to two light flavors. We first keep $T$ fixed, verifying that the expected periodicity of the different thermodynamical quantities as functions of $\theta$ is indeed satisfied. Moreover, for $T>T_{R W}$ we find the mentioned RW first order phase transition at $\theta=\pi / 3$, which is signalled by a discontinuity in the phase of the Polyakov loop field. The values obtained for $T_{R W}$ are $191 \mathrm{MeV}, 188 \mathrm{MeV}$ and $191 \mathrm{MeV}$ for sets A, B and C, respectively, in good agreement with the lattice QCD estimate $T_{R W}=185$ (9) [28]. Concentrating on the sector $0 \leqslant \theta \leqslant \pi / 3$ we observe that for values of the temperature $T_{c}(\theta=0) \leqslant T \leqslant T_{R W}$ the order parameters for both deconfinement and chiral symmetry show signals of a phase transition at a given value of $\theta$. This is clearly seen in Fig. 3 where we plot the behavior of order parameters and susceptibilities as functions of $T$ taking now $\theta$ fixed at two representative values $\theta=\pi / 6$ and $\pi / 3$. The plots correspond to parameter set $C$. We note that while for $\theta=\pi / 6$ both deconfinement and chiral restoration are crossover-like, they are first order for $\theta=\pi / 3$. As in the case of $\theta=0$ (see the curves corresponding to $T_{0}=208 \mathrm{MeV}$ in Fig. 1), for both values of $\theta$ the deconfinement and chiral restoration transitions occur at the same temperature, given by the peaks of the susceptibilities or the positions of the discontinuities. Although one might argue that there is a certain tendency of the chiral susceptibility to decay more slowly or even, in the case of $\theta=\pi / 3$, to display a very broad peak on the high temperature side, one can hardly conclude that both transitions apart from
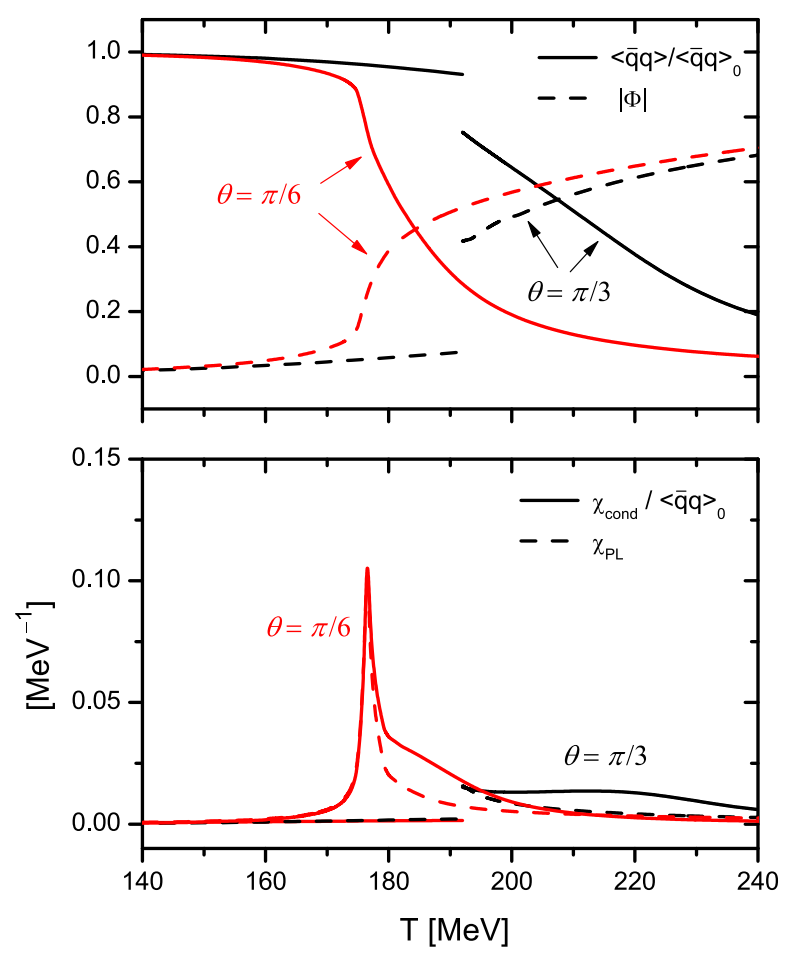

Fig. 3. Order parameters for the deconfinement and chiral restoration transitions (upper panel) and corresponding susceptibilities (lower panel) as functions of $T$ for $\theta=\pi / 6$ and $\theta=\pi / 3$. Curves correspond to set $\mathrm{C}$.

each other more than about $20 \mathrm{MeV}$, even for $\theta=\pi / 3$. The situation is quite similar for parameter set $B$ (which also includes WFR), whereas for set $A$ one gets just first order transitions for all values of $\theta$ in the range of temperatures considered. The dependence of the critical temperature $T_{c}$ as a function of $\theta$ is shown in Fig. 4 for our three parameter sets. For comparison, we also show the corresponding lattice results given in Ref. [28], which include an error of about $10 \%$ due to the uncertainty in the lattice determination of $T_{c}(\theta=0)$. As already mentioned, while for set $A$ both the deconfinement and chiral restoration transitions are always first order, in the case of sets $B$ and $C$ there is a critical value $\theta_{C E P} \sim 0.7 \times \pi / 3$ below which the transitions become crossover-like. Thus, we find that for all three parameterizations the corresponding transition lines are first order when they meet the RW endpoint. This implies that the RW endpoint is a triple point, the RW transition being also first order there. The character of the RW transition at the RW endpoint is clearly seen in Fig. 5, where we plot the behavior of the phase of the extended Polyakov loop $\Psi$ at $\theta=\pi / 3$ as a function of $T$ (the figure corresponds to set C). The extended Polyakov loop, defined by $\Psi=\exp (i \theta) \Phi$, is by construction invariant under the transformations in Eq. (12), and its phase $\psi$ can be taken as order parameter of the RW transition [42].

Finally, it is interesting to analyze the behavior of $\chi_{\text {cond }}=$ $d\langle\bar{q} q\rangle / d T$ in comparison with that of the more conventional chiral susceptibility $\chi_{\mathrm{ch}}=d\langle\bar{q} q\rangle / d m_{q}$. Following Refs. [22-25] we have so far, for simplicity, used the first one. However, as argued in Ref. [47], different observables might behave differently in the crossover region. In fact, especially at real finite chemical potential, the $\mu$ dependence of the splittings between the transition temperatures obtained from different observables might serve as an indicator of the existence and location of a critical end point (CEP). To illustrate our results we display in Fig. 6 the behavior of $\chi_{\text {cond }}, \chi_{\text {ch }}$ and $\chi_{\Phi}$ for the case of set $C$, considering temperature ranges of $30 \mathrm{MeV}$ around the position of the corresponding peaks. 

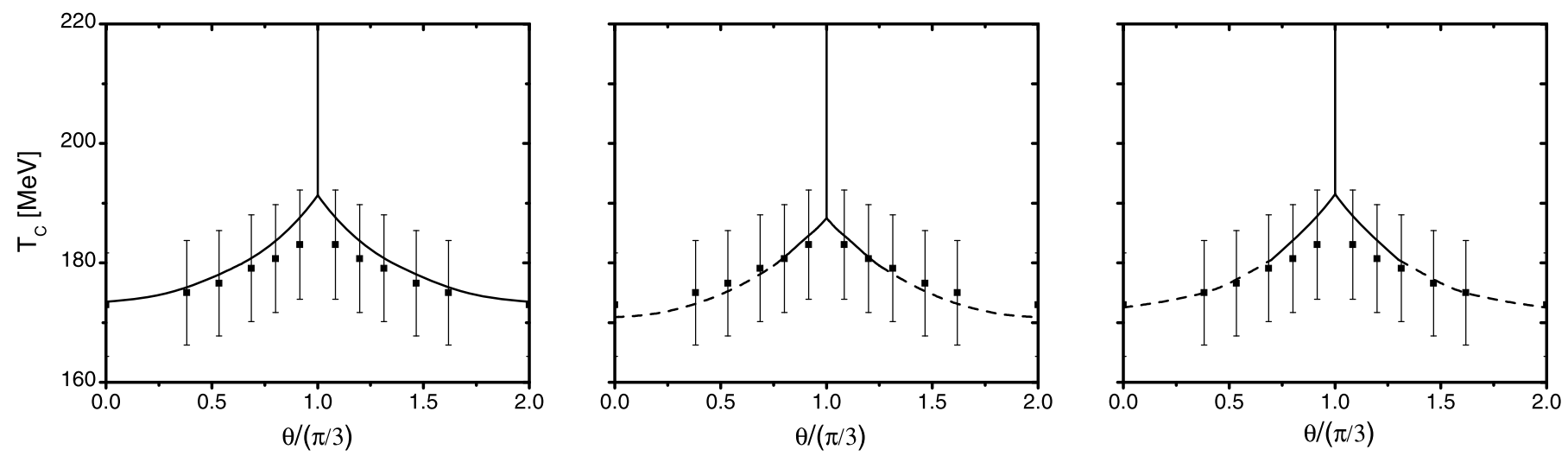

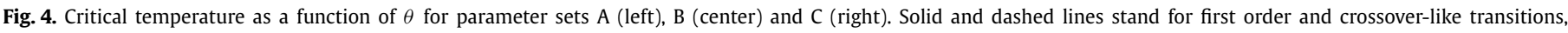
respectively. Dots correspond to lattice QCD results. Vertical solid lines correspond to the first order RW transition.

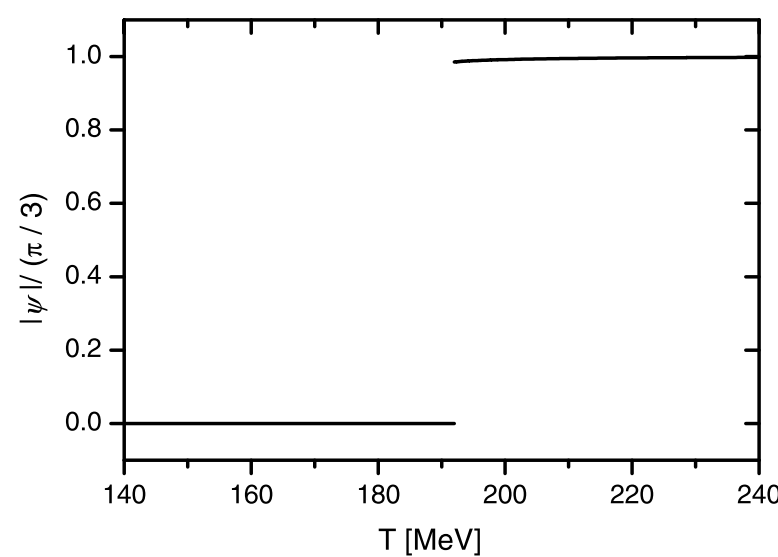

Fig. 5. Phase of the extended Polyakov loop $\Psi=\exp (i \theta) \Phi$ as a function of $T$ for $\theta=\pi / 3$. Curves correspond to set $\mathrm{C}$.

We take several representative values for the chemical potential, in the regions of both imaginary and real $\mu$. As in the case of the model with $T_{0}=270 \mathrm{MeV}$, previously studied in Ref. [31], one also finds a CEP in the region of finite real chemical potential. For $T_{0}=$ $208 \mathrm{MeV}$ this point is located at $(\mu, T)=(213 \mathrm{MeV}, 140 \mathrm{MeV})$, while in the imaginary $\mu$ region the value of $\theta_{\text {CEP }}$ given above leads to a CEP located at $(\mu, T)=(132 i \mathrm{MeV}, 180 \mathrm{MeV})$. The upper left panel and the lower right panel in Fig. 6 correspond to values of $\mu$ close to these critical endpoints. In general, though not distinguishable in the figure, after a detailed analysis one can find small differences in the positions of the peaks of the susceptibilities. At $\mu=0$ the peak of $\chi_{\text {cond }}$ is found to be about $0.02 \mathrm{MeV}$ above that of $\chi_{\mathrm{ch}}$, while the peak of $\chi_{\Phi}$ is located $0.05 \mathrm{MeV}$ below. These values are only a few times larger than our numerical uncertainty, that we estimate to be about $0.01 \mathrm{MeV}$. As one gets into the region of finite real $\mu$ we first observe an enhancement of the splittings. This happens up to $\mu \simeq 130 \mathrm{MeV}$, where we find that the peaks of $\chi_{\text {cond }}$ and $\chi_{\Phi}$ are located about $0.06 \mathrm{MeV}$ and $0.20 \mathrm{MeV}$ below that of $\chi_{\mathrm{ch}}$, respectively. However, as $\mu$ increases further towards the CEP we find that the splittings get reduced, a behavior that can be taken as a signal of the presence of the CEP. For example, at $\mu=180 \mathrm{MeV}$ the splittings get reduced by a factor of $1 / 3$ in comparison with the values at $\mu=130 \mathrm{MeV}$, and already at $\mu=210 \mathrm{MeV}$ (i.e. $3 \mathrm{MeV}$ below the CEP) we find that both splittings vanish within our level of uncertainty. In the region of imaginary chemical potential we find that the separations between the peaks of the different susceptibilities always decrease from their values at $\mu=0$, and once again become zero as one ap- proaches the corresponding CEP. We have also performed the analysis for the simpler exponential parameterization set B. Though in this case the splittings are even smaller than in the case of set $C$, the faster convergence of the integrals and sums allows a better numerical precision. The behavior of the splittings as functions of $\mu$ is qualitatively similar to the one described above, both in the real and imaginary chemical potential regions. In particular, they vanish as one approaches the critical endpoints. Finally, we notice that in the region of imaginary chemical potential the already mentioned tendency of the peaks of $\chi_{\text {cond }}$ to become broader in the high temperature side is somewhat stronger in the case of the chiral susceptibility. In fact, $\chi_{\text {ch }}$ shows a widening that includes a second low, broad peak. The separation between this second peak and the main peak is found to be about $14 \mathrm{MeV}$ in the vicinity of the CEP, becoming smaller as $\mu$ approaches to zero (e.g. both peaks are separated by $11 \mathrm{MeV}$ and $7 \mathrm{MeV}$ for $\mu=100 i \mathrm{MeV}$ and $\mu=50 i \mathrm{MeV}$, respectively). The second broad peak in $\chi_{\mathrm{ch}}$ is not observed in the region of real $\mu$ (see figure).

In summary, we have considered the impact of the feedback of the dynamical quarks on the parameter $T_{0}$ appearing in the Polyakov potential, as proposed in Ref. [23]. This has been done here in the context of non-local PNJL models, considering three different types of non-local form factors. We have studied the deconfinement and chiral restoration transitions, determining the corresponding critical temperatures and the character of the transitions. The results are found to be in agreement with those obtained in lattice QCD, showing a strong entanglement between both critical temperatures for zero and imaginary chemical potential. In our context this arises as a consequence of the natural dependence of effective quark interactions on the Polyakov loop through the appearance of the background color field in the argument of nonlocal form factors. The determination of the critical temperatures in the crossover region has been carried out by analyzing different susceptibilities. It is seen that in general there is a small splitting between the positions of the corresponding peaks, which tends to vanish as one approaches the critical endpoints, both in the region of real and imaginary chemical potential. Finally, we have also analyzed the Roberge-Weiss transition, which is found to be first order at the RW endpoint for all three parameter sets.

\section{Acknowledgements}

This work has been partially supported by CONICET (Argentina) under grants \# PIP 00682 and PIP 02495, and by ANPCyT (Argentina) under grant \# PICT07 03-00818. 

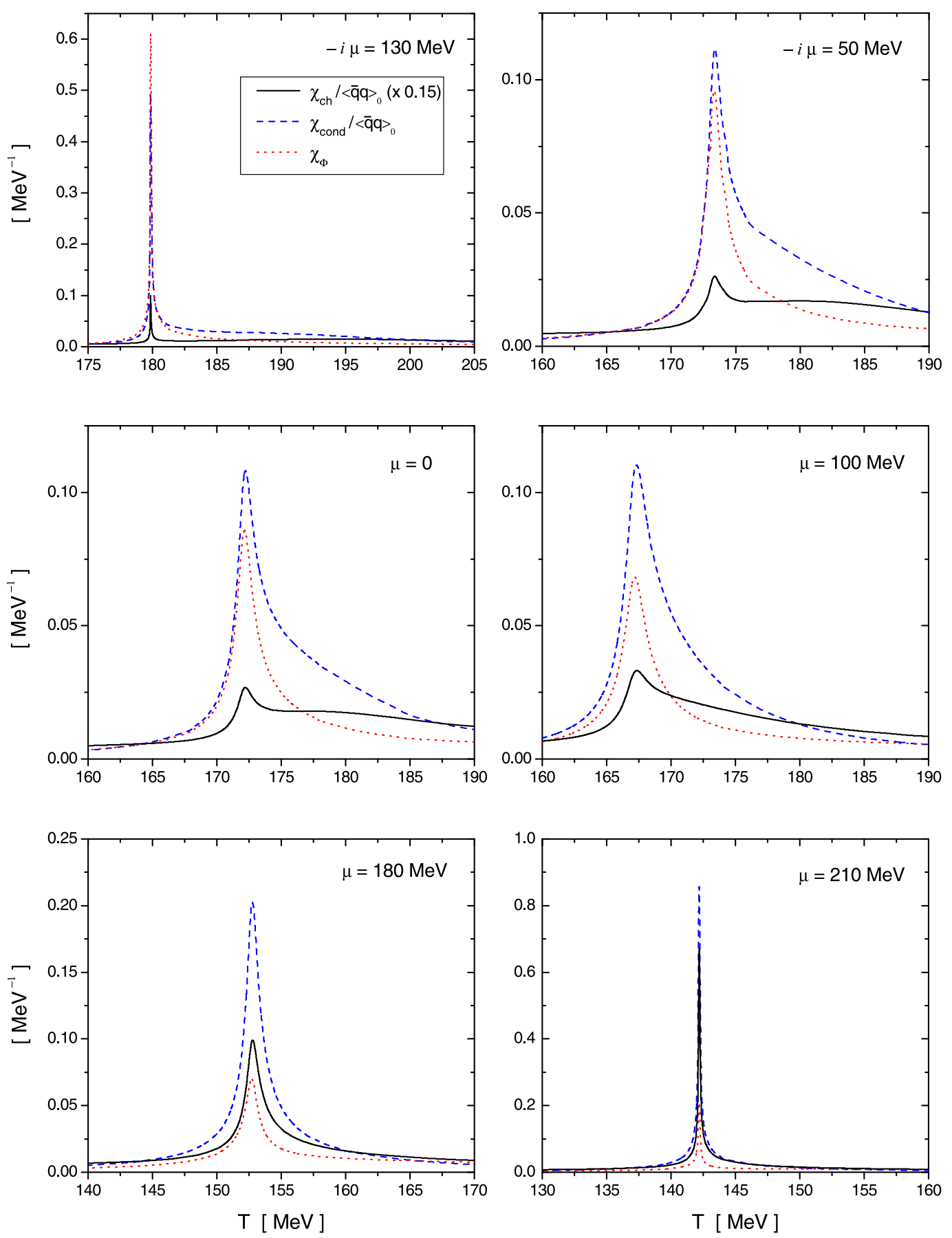

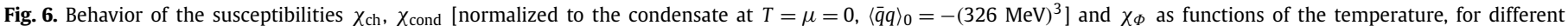
representative values of the chemical potential. Results correspond to set $C$.

\section{References}

[1] See e.g.: D.H. Rischke, Prog. Part. Nucl. Phys. 52 (2004) 197; P. Jacobs, X.N. Wang, Prog. Part. Nucl. Phys. 54 (2005) 443; K. Fukushima, T. Hatsuda, arXiv:1005.4814 [hep-ph].

[2] C.R. Allton, et al., Phys. Rev. D 68 (2003) 014507; C.R. Allton, et al., Phys. Rev. D 71 (2005) 054508.

[3] Z. Fodor, S.D. Katz, JHEP 0404 (2004) 050; Y. Aoki, Z. Fodor, S.D. Katz, K.K. Szabo, JHEP 0601 (2006) 089.

[4] F. Karsch, E. Laermann, in: R.C. Hwa, X.N. Wang (Eds.), Quark Gluon Plasma III, World Scientific, Singapore, 2004, arXiv:hep-lat/0305025.

[5] P.N. Meisinger, M.C. Ogilvie, Phys. Lett. B 379 (1996) 163.

[6] K. Fukushima, Phys. Lett. B 591 (2004) 277.

[7] E. Megias, E. Ruiz Arriola, L.L. Salcedo, Phys. Rev. D 74 (2006) 065005.

[8] C. Ratti, M.A. Thaler, W. Weise, Phys. Rev. D 73 (2006) 014019.
[9] S. Roessner, C. Ratti, W. Weise, Phys. Rev. D 75 (2007) 034007.

[10] S. Mukherjee, M.G. Mustafa, R. Ray, Phys. Rev. D 75 (2007) 094015.

[11] C. Sasaki, B. Friman, K. Redlich, Phys. Rev. D 75 (2007) 074013.

[12] U. Vogl, W. Weise, Prog. Part. Nucl. Phys. 27 (1991) 195;

S. Klevansky, Rev. Mod. Phys. 64 (1992) 649;

T. Hatsuda, T. Kunihiro, Phys. Rep. 247 (1994) 221;

M. Buballa, Phys. Rep. 407 (2005) 205.

[13] D. Blaschke, M. Buballa, A.E. Radzhabov, M.K. Volkov, Yad. Fiz. 71 (2008) 2012, Phys. At. Nucl. 71 (2008) 1981.

[14] G.A. Contrera, D. Gómez Dumm, N.N. Scoccola, Phys. Lett. B 661 (2008) 113; G.A. Contrera, D. Gómez Dumm, N.N. Scoccola, Phys. Rev. D 81 (2010) 054005.

[15] T. Hell, S. Roessner, M. Cristoforetti, W. Weise, Phys. Rev. D 79 (2009) 014022; T. Hell, S. Roessner, M. Cristoforetti, W. Weise, Phys. Rev. D 81 (2010) 074034; T. Hell, K. Kashiwa, W. Weise, arXiv:1104.0572 [hep-ph]: K. Kashiwa, arXiv:1104.5167 [hep-ph]. 
[16] S. Noguera, N.N. Scoccola, Phys. Rev. D 78 (2008) 114002.

[17] R.D. Bowler, M.C. Birse, Nucl. Phys. A 582 (1995) 655; R.S. Plant, M.C. Birse, Nucl. Phys. A 628 (1998) 607.

[18] W. Broniowski, B. Golli, G. Ripka, Nucl. Phys. A 703 (2002) 667; A.H. Rezaeian, N.R. Walet, M.C. Birse, Phys. Rev. C 70 (2004) 065203.

[19] A. Scarpettini, D. Gómez Dumm, N.N. Scoccola, Phys. Rev. D 69 (2004) 114018.

[20] D. Gómez Dumm, A.G. Grunfeld, N.N. Scoccola, Phys. Rev. D 74 (2006) 054026.

[21] K.-I. Kondo, Phys. Rev. D 82 (2010) 065024; K.-I. Kondo, arXiv:1103.3829 [hep-th].

[22] D. Horvatic, D. Blaschke, D. Klabucar, O. Kaczmarek, arXiv:1012.2113 [hep-ph].

[23] B.-J. Schaefer, J.M. Pawlowski, J. Wambach, Phys. Rev. D 76 (2007) 074023; B.-J. Schaefer, M. Wagner, J. Wambach, Phys. Rev. D 81 (2010) 074013 T.K. Herbst, J.M. Pawlowski, B.J. Schaefer, Phys. Lett. B 696 (2011) 58.

[24] Y. Sakai, K. Kashiwa, H. Kouno, M. Matsuzaki, M. Yahiro, Phys. Rev. D 79 (2009) 096001.

[25] Y. Sakai, T. Sasaki, H. Kouno, M. Yahiro, Phys. Rev. D 82 (2010) 076003.

[26] M. D’Elia, M.-P. Lombardo, Phys. Rev. D 67 (2003) 014505.

[27] P. de Forcrand, O. Philipsen, Nucl. Phys. B 642 (2002) 290.

[28] L.-K. Wu, X.-Q. Luo, H.-S. Chen, Phys. Rev. D 76 (2007) 034505.

[29] J. Braun, L.M. Haas, F. Marhauser, J.M. Pawlowski, Phys. Rev. Lett. 106 (2011) 022002.
[30] A. Roberge, N. Weiss, Nucl. Phys. B 275 (1986) 734.

[31] G.A. Contrera, M. Orsaria, N.N. Scoccola, Phys. Rev. D 82 (2010) 054026.

[32] D. Gómez Dumm, N.N. Scoccola, Phys. Rev. C 72 (2005) 014909.

[33] S. Borsanyi, Z. Fodor, C. Hoelbling, S.D. Katz, S. Krieg, C. Ratti, K.K. Szabo, Wuppertal-Budapest Collaboration, JHEP 1009 (2010) 073.

[34] F. Karsch, E. Laermann, Phys. Rev. D 50 (1994) 6954.

[35] C.W. Bernard, et al., Phys. Rev. Lett. 78 (1997) 598.

[36] Y. Iwasaki, K. Kanaya, S. Kaya, T. Yoshie, Phys. Rev. Lett. 78 (1997) 179.

[37] S. Aoki, et al., JLQCD Collaboration, Phys. Rev. D 57 (1998) 3910.

[38] A. Ali Khan, et al., CP-PACS Collaboration, Phys. Rev. D 63 (2001) 034502.

[39] M. D'Elia, A. Di Giacomo, C. Pica, Phys. Rev. D 72 (2005) 114510.

[40] C. Bonati, G. Cossu, M. D’Elia, A. Di Giacomo, C. Pica, PoS LATTICE2008 (2008) 204.

[41] R.D. Pisarski, F. Wilczek, Phys. Rev. D 29 (1984) 338.

[42] Y. Sakai, K. Kashiwa, H. Kouno, M. Yahiro, Phys. Rev. D 77 (2008) 051901; Y. Sakai, K. Kashiwa, H. Kouno, M. Yahiro, Phys. Rev. D 78 (2008) 036001.

[43] M. D’Elia, F. Di Renzo, M.P. Lombardo, Phys. Rev. D 76 (2007) 114509.

[44] M. D’Elia, F. Sanfilippo, Phys. Rev. D 80 (2009) 111501.

[45] P. de Forcrand, O. Philipsen, Phys. Rev. Lett. 105 (2010) 152001.

[46] C. Bonati, G. Cossu, M. D'Elia, F. Sanfilippo, arXiv:1011.4515 [hep-lat].

[47] G. Endrodi, Z. Fodor, S.D. Katz, K.K. Szabo, JHEP 1104 (2011) 001. 